\begin{tabular}{|c|l|}
\hline Title & P metabolism and transport in AM fungi \\
\hline Author(s) & Ezawa, Tatsuhiro; Smith, Sally E.; Smith, F. A ndrew \\
\hline Citation & $\begin{array}{l}\text { Plant and Soil, 244(1-2), 221-230 } \\
\text { https://doi.org/10.1023/A:1020258325010 }\end{array}$ \\
\hline Issue Date & 2002-07 \\
\hline Doc URL & http://hdl.handle.net/2115/637 \\
\hline Rights & The original publication is available at www.springerlink.com \\
\hline Type & article (author version) \\
\hline File Information & EzPLSO.pdf \\
\hline
\end{tabular}

Instructions for use 
Title: $\quad$ P metabolism and transport in AM fungi

Authors: $\quad$ Tatsuhiro Ezawa ${ }^{1}$, Sally E. Smith ${ }^{2}$, and F. Andrew Smith ${ }^{2}$

Affiliations: $\quad{ }^{1}$ University Farm, Nagoya University, Togo-cho, Aichi 470-0151 Japan.

${ }^{2}$ Department of Soil and Water and Centre for Plant Root Symbioses, Waite,

Campus, Adelaide University, Glen Osmond, South Australia, 5064, Australia.

Short title: $\quad$ P metabolism and transport in AM fungi

No. of pages: 22

No. of figures: 1

No. of tables: Nil

\section{Corresponding author:}

Tatsuhiro Ezawa

University Farm, Nagoya University, Togo-cho, Aichi 470-0151 Japan

TEL: +81-5613-7-0207, FAX: +81-5613-8-4473

E-mail: tatsu@agr.nagoya-u.ac.jp 


\title{
$P$ metabolism and transport in AM fungi
}

Tatsuhiro Ezawa ${ }^{1}$, Sally E. Smith², and F. Andrew Smith ${ }^{2}$.

${ }^{1}$ University Farm, Nagoya University, Togo-cho, Aichi 470-0151 Japan

${ }^{2}$ Department of Soil and Water and Centre for Plant Root Symbioses, Waite, Campus, Adelaide University, Glen Osmond, South Australia, 5064, Australia.

Key words: arbuscular mycorrhizal fungi, P metabolism, P transport, polyphosphate, V-ATPase, vacuoles.

\begin{abstract}
The arbuscular mycorrhizal symbiosis is mutualistic, based on reciprocal transfer of $\mathrm{P}$ from the fungus to the plant and carbon from the plant to the fungus. Thus P is a most important 'currency' in the symbiosis. After absorbing P from the soil solution, the fungi first incorporate it into the cytosolic pool, and the excess P is transferred to the vacuoles. The vacuolar P pool probably plays a central role in $\mathrm{P}$ supply to the plant. The main forms of inorganic $\mathrm{P}$ in fungal vacuoles are orthophosphate and polyphosphate, but organic P molecules may also be present. Long distance translocation of $\mathrm{P}$ from the site of uptake in the external mycelium to the site of transfer to the plant is probably achieved via transfer of vacuolar components. This transport would be mediated either by protoplasmic streaming or the motile tubular vacuole-like system. The site of release of P into the interfacial apoplast and thence to the plant is most probably the fungal arbuscules. The biochemical and biophysical processes involved in P metabolism and transfer between cellular compartments in the symbiosis are currently not well understood. Some recent investigations of substrate specificities of phosphatase-type enzymes in AM fungi and other eukaryotic microorganisms, however, have shed new light on earlier results and permit the construction of a
\end{abstract}


hypothetical scheme of P-flow, including possible regulatory factors. Steps in this scheme are experimentally testable and should stimulate future research.

\section{Introduction}

Arbuscular mycorrhizal (AM) fungi colonize the root cortex of the host plant in which the fungi are able to acquire organic $\mathrm{C}$ to build 'the infrastructure' for $\mathrm{P}$ uptake and transport. The mycorrhizal system is able to take up P more efficiently and transport P over longer distances than the plant root system, overcoming P depletion in the rhizosphere (Zhu et al., 2001). Accordingly, mechanisms of P metabolism and transport are of great interest, not only from the view-point of basic biology but also from that of applied science. Biochemical processes in the fungi are, however, difficult to investigate because the fungi are obligate biotrophs. Targeted molecular biological approaches are particularly important to unravel the nature of AM fungi, including information on the expression of genes encoding proteins involved in P uptake, turnover, transport and transfer to the plant. However, for this strategy we need to be in possession of the necessary 'targets'; i.e. we need a testable model of possible pathways of $\mathrm{P}$ metabolism. In this review we attempt to integrate the latest information on P metabolism and transport in AM fungi with that for other eukaryotes, to give a whole picture of the system in order to stimulate future targeted approaches.

\section{Fungal $P$ uptake, compartmentation and $P$ pools}

There is a considerable concentration gradient of inorganic orthophosphate (Pi) between the soil solution and plant/fungal cytoplasm. In the former concentrations rarely exceed $10 \mu \mathrm{M}$, whereas in plants the cytoplasmic concentration is probably around $10 \mathrm{mM}$ (Mimura, 1999). To absorb Pi under these conditions, plants and soil microorganisms have developed specialized, energy dependent systems for Pi uptake. Both high-affinity (low $K_{\mathrm{m}}$ and $V_{\max }$ ) and low-affinity (high $K_{\mathrm{m}}$ and $V_{\max }$ ) transport systems have been identified and include $\mathrm{H}^{+} / \mathrm{Pi}$ symporters, with the necessary proton gradient produced by plasma membrane $\mathrm{H}^{+}$-ATPases (P-ATPases). Typical $K_{\mathrm{m}}$ values of 
high-affinity systems in plants (Mimura, 1999) and fungi (Beever and Burns, 1980) are in the range from 1 to $10 \mu \mathrm{M}$, and it might be predicted that those of AM fungi would be within this range. However, values of the $K_{\mathrm{m}} \mathrm{s}$ in $\mathrm{AM}$ fungi vary considerably, depending on the methods used. Thomson et al. (1990) showed that for germ tubes of Gigaspora margarita, the $K_{\mathrm{m}}$ of the high-affinity and low-affinity systems were 1.8-3.1 $\mu \mathrm{M}$ and 10.2-11.3 mM, respectively. However, a $K_{\mathrm{m}}$ value of the high-affinity system of extraradical hyphae of AM fungi estimated from physiological-P uptake data in a soil culture system was about one order of magnitude lower, $0.17 \mu \mathrm{M}$ (Schweiger and Jakobsen, 1999) and that of GvPT, a high-affinity Pi transporter expressed in the external mycelium of the AM fungus Glomus versiforme was much higher, $18 \mu \mathrm{M}$ (Harrison and van Buuren, 1995). The high value may be an artefact of the yeast complementation system used to test Pi transporter function for GvPT (see Versaw this volume). Additional information on the Pi uptake system can be derived from measurements of the transmembrane potential difference (PD) of germ tubes and extraradical hyphae. Values are rather low [around $-47 \mathrm{mV}$, (Ayling et al., 2000)], compared with other organisms, but using this value and an external Pi concentration of 35 $\mu \mathrm{M}$ gives an $\mathrm{H}^{+} / \mathrm{H}_{2} \mathrm{PO}_{4}{ }^{-}$stoichiometry of 2.33 . This is higher than the most generally accepted ratio of 2.0, but within the range of published values. However, the much lower $K_{\mathrm{m}}$ for hyphal uptake from soil (c. $0.2 \mu \mathrm{M}$; see above) poses energetic problems for this stoichiometry because it shows that there is Pi uptake against very large electrochemical potential gradients.

Another high-affinity system, a $\mathrm{Na}^{+}$-coupled Pi transporter, is known from animals, bacteria (Reizer et al., 1994) and plants (Reid et al., 2000) and has also been found in eukaryotic microorganisms including yeast, Saccharomyces cerevisiae, and the filamentous fungus, Neurospora crassa. These two fungi possess both $\mathrm{H}^{+} / \mathrm{Pi}$ and $\mathrm{Na}^{+} / \mathrm{Pi}$ co-transport systems, and the genes encoding these transporters have been identified (Martinez and Persson, 1998; Versaw and Metzenberg, 1995). In the case of yeast, both the $\mathrm{H}^{+} / \mathrm{Pi}$ and $\mathrm{Na}^{+} / \mathrm{Pi}$ transporter genes are controlled in the PHO pathway (Martinez and Persson, 1998) which promotes gene transcription in response to Pi deficiency (Lenburg and O'Shea, 1996). The physiological role of these transporters can be 
interpreted from their different $\mathrm{pH}$ optima: the $\mathrm{H}^{+} / \mathrm{Pi}$ system operates optimally at neutral $\mathrm{pH}$ and the $\mathrm{Na}^{+} / \mathrm{Pi}$ system at alkaline $\mathrm{pH}$, suggesting that the organisms can take up Pi efficiently with the two systems over a broad pH range (Martinez and Persson, 1998). It is possible that AM fungi possess a $\mathrm{Na}^{+} / \mathrm{Pi}$ transport system as well as the $\mathrm{H}^{+} / \mathrm{Pi}$ system, which would contribute to $\mathrm{Pi}$ acquisition from alkaline soil.

Following uptake, Pi is first incorporated into the cytosolic Pi pool with the concentration kept constant to maintain various cell functions, such as energy generation and biosynthesis of phospholipid, nucleic acid and precursors of carbohydrate polymers such as UDP-glucose. If the fungi are able to take up more Pi than the amount required for maintenance of cell functions, the excess is transported into the vacuoles, effectively buffering the cytosolic Pi concentration (Klionsky et al., 1990; Shirahama et al., 1996). It has been demonstrated that Pi transport into plant vacuoles depends on an electrical potential gradient (not a chemical gradient for $\mathrm{H}^{+}$) created by a vacuolar $\mathrm{H}^{+}$-ATPase (V-ATPase) (Massonneau et al., 2000). In the case of yeast, Booth and Guidotti (1997) demonstrated that isolated vacuoles took up a large amount of Pi by exchange with Pi present in the vacuoles i.e. the Pi uptake was driven by the counterflow of Pi. Indeed, the Pi uptake was considerably reduced in the absence of $\mathrm{Pi}$ in the vacuoles. This could not be an acceptable mechanism for net influx unless the stoichiometry was other than 1:1. On the other hand, Kulakovskaya and Kulaev (1997) observed unidirectional transport of Pi into isolated yeast vacuoles, with uptake showing linear kinetics with respect to external Pi concentration up to $10 \mathrm{mM}$. Both the systems found in yeast were insensitive to protonophoric uncouplers, indicating that the systems were independent of the $\mathrm{H}^{+}$gradient. However, it is uncertain if membrane potential, the electric component of the electrochemical gradient created by V-ATPase, is involved in the fungal vacuolar transport, and the transport mechanism is unresolved. In fungi, it may be important that polyphosphate synthesis (described in the next section) coincides with Pi uptake during $\mathrm{P}$ accumulation in the vacuole to prevent large-scale efflux. In the case of AM fungi, this vacuolar pool may play a central role in P supply to the host plant. 


\section{$\mathbf{P}$ metabolism in vacuoles}

The vacuole is a most fascinating organelle in the context of symbiotic P metabolism and transport in AM fungi. Whereas most biochemical reactions occurring in the cytosol may be essential for survival/growth of AM fungi and occur in common with other fungi, the vacuoles could, in addition, be organized as unique regulatory systems, important for the establishment of the mutualistic relationship with plants.

\section{Inorganic $P$}

Inorganic $\mathrm{P}$ is a major component of the vacuolar $\mathrm{P}$ pool of AM fungi, with contributions from both $\mathrm{Pi}$ and polyphosphate (polyP). PolyP was first visualized in the vacuole as electron-dense and P-rich 'granules' and suggested to be the main form of $\mathrm{P}$ transported over long distances in these fungi (Callow et al., 1978; Cox et al., 1980). PolyP is a linear polymer of Pi residues linked by high-energy phosphoanhydride bonds. Although various cellular functions have been suggested (Kornberg et al., 1999), the most important role in fungi is probably as a temporary storage of Pi. The chain-length of polyP in AM fungi is variable (Ezawa et al., 1999), being longer in extraradical than in intraradical hyphae (Solaiman et al., 1999). This may reflect differences in polyP metabolism between the two phases (Ezawa et al., 2001a). Both soluble and insoluble (granular) forms of polyP have been found in nature (Clark et al., 1986). The status of granular polyP in an ectomycorrhizal fungus, Pisolithus tinctorius has been questioned. It has been suggested that the granules seen by electron microscopy in the fungus are an artefact produced during the fixation of tissue and that polyP is always dispersed throughout the vacuoles in soluble form (Orlovich and Ashford, 1993). However, Bücking and Heyser (1999) have observed granular polyP in P. tinctorius. The matter remains unresolved. Clark et al. (1986) have established a successive extraction method in which short-chain soluble, long-chain soluble and long-chain granular polyP can be fractionated. Using this method, polyP was shown to occur in both soluble and long-chain granular forms in the 
intra- and extraradical hyphae of the AM fungus Gigaspora margarita (Solaiman et al., 1999). Many factors may affect solubility of polyP in vivo, including $\mathrm{pH}$, chain-length, concentration of polyP and counter ions such as metal cations and polyamines (Cramer and Davis, 1984).

Accordingly, the form of polyP in vivo may be quite varied among species and also in the same species grown under different environmental conditions.

PolyP metabolism is best understood from work on prokaryotes, in which many enzymes have been found and characterized (Kornberg et al., 1999). In prokaryotes polyphosphate kinase (PPK) synthesizes polyP using ATP as a phosphoryl donor and also catalyzes a reverse reaction (ATP regeneration from polyP). Polyphosphate glucokinase (PPGK) transfers a terminal Pi residue to glucose and synthesizes glucose 6-phosphate. Exo- and endopolyphosphatases (PPX and PPN) hydrolyze terminal and internal linkages, respectively. The following diagram summarises the catalytic reactions of these enzymes.

\section{Phosphotransferase type}

PPK: $\quad$ polyP $_{n-1}+\mathrm{ATP} \rightarrow$ polyP $_{n}+\mathrm{ADP}$

PPGK: $\quad$ polyP $_{\mathrm{n}}+$ glucose $\rightarrow$ polyP $_{\mathrm{n}-1}+$ glucose-6-phosphate

\section{Phosphatase type}

PPX: $\quad \operatorname{polyP}_{n}+\mathrm{H}_{2} \mathrm{O} \rightarrow$ polyP $_{\mathrm{n}-1}+\mathrm{Pi}$

PPN: $\quad \operatorname{polyP}_{n}+\mathrm{H}_{2} \mathrm{O} \rightarrow \operatorname{polyP}_{n-m}+\operatorname{polyP}_{m}(\mathrm{~m} \leq 3)$

The presence of similar enzymes in eukaryotes and their roles in vacuolar P metabolism is not well understood. Capaccio and Callow (1982), pioneer researchers on the polyP metabolism of AM fungi, showed the activities of all of the above enzymes in G. mosseae. However, PPK and PPGK have not been demonstrated in any other eukaryotic microorganisms (Booth and Guidotti, 1995; Kornberg et al., 1999). Recently, we attempted to repeat the work of Capaccio and Callow (1982) but failed to detect either PPK (Ezawa unpublished results) or PPGK (Ezawa et al., 2001b) activity in the AM 
fungi Glomus coronatum and G. etunicatum. It is mysterious that PPK, the only enzyme which is involved in polyP synthesis, is apparently missing in the eukaryotes investigated, even in yeast which accumulates polyP up to $10-20 \%$ of the dry weight (Kornberg et al., 1999). However, a homologue of the bacterial PPK gene has been found in the slime mould, Dictyostelium. The gene was responsible for antifungal drug-resistance and the actual function of the gene product in polyP metabolism is yet to be characterized (Kornberg et al., 1999). Ogawa et al. (2000) found four genes involved in polyP accumulation in yeast and hypothesized that polyP might be synthesized through a system analogous to the mitochondrial (F-type) $\mathrm{H}^{+}$-ATPase, which generates ATP by mechanism energized by the proton gradient. According to their observations, a protein complex translated from the genes, which was apparently different from V-ATPase, might be localized on the yeast tonoplast and be responsible for polyP synthesis, energized in this case by a proton gradient built by V-ATPase. PolyP in AM fungi could be synthesized by a similar system.

The presence of several PPXs has been found for different cell compartments of yeast: the cell envelope (Andreeva and Okorokov, 1993), cytosol (Andreeva et al., 1998a; Wurst and Kornberg, 1994), vacuoles (Andreeva et al., 1998b), nuclei (Lichko et al., 1996) and mitochondria (Lichko et al., 1998). These enzymes showed different pH optima, substrate (chain-length) specificity and sensitivity to various effectors (Kulaev et al., 1997). The cytosolic PPX has been purified and well characterized. It showed a $\mathrm{pH}$ optimum at 7.5, specificity for long-chain polyP and was inhibited by polyamine. The only eukaryotic gene involved in polyP metabolism cloned to date is that encoding this enzyme (Wurst et al., 1995). Two types of PPX activity have been found in the AM fungi, Glomus coronatum and G. etunicatum (Ezawa et al., 2001a). One had a pH optimum near neutrality and exhibited a low $K_{\mathrm{m}}$ with long-chain polyP, similar to the yeast cytosolic PPX (Andreeva et al., 1998a; Wurst and Kornberg, 1994). The other activity showed an acidic $\mathrm{pH}$ optimum and a low $K_{\mathrm{m}}$ with short-chain polyP, similar to that found in $G$. mosseae by Capaccio and Callow (1982). This activity was characterized as non-specific acid phosphatase (ACPase) and may be localized in the vacuoles (see below). PPN has been found in various fungi 
(Kumble and Kornberg, 1996) including AM fungi (Capaccio and Callow, 1982), but only yeast PPN has been purified from the vacuole and characterized (Kumble and Kornberg, 1996). In this case the main product of polyP hydrolysis was tripolyphosphate, with an optimum $\mathrm{pH}$ of 7.5. In AM fungal symbiosis it seems likely that PPX, ACPase and PPN would work together to hydrolyze polyP into Pi completely.

One interesting but very different hypothesis with respect to PPX is its possible function as a Pi pump (transporter) in vacuoles and lysosomes. Kulaev and Kulakovskaya (2000) proposed that cytosolic Pi is taken up through PPX bound to the tonoplast and incorporated directly into polyP by the enzyme. The hypothesis was based upon the observation that ${ }^{32} \mathrm{P}$-labled Pi was rapidly incorporated into a long-chain polyP in isolated human fibroplast lysosomes (Pisoni and Lindley, 1992). However, the way in which energy is supplied for the polymerization remains obscure.

\section{Organic $P$}

Regulation of cell function and activity requires tight control of the concentration of metabolites, including various P-esters, and surpluses are transported into vacuoles and metabolized there (Klionsky et al., 1990). Among hydrolase-type enzymes found in fungal vacuoles, phosphatases are responsible for the conversion of P-esters into Pi. Non-specific alkaline and acid phosphatases (ALPase and ACPase, respectively) have been demonstrated in AM fungi by electrophoresis (Fabig et al., 1989; Gianinazzi-Pearson and Gianinazzi, 1978), biochemical (Ezawa et al., 1999), histochemical (Ezawa et al., 1995; Tisserant et al., 1993) and cytochemical (Gianinazzi et al., 1979) studies. Both the ALPase and ACPase have been observed in vacuoles of the fungi (Gianinazzi et al., 1979), and most of the activity of these enzymes was associated with the insoluble fraction (Ezawa et al., 1999), possibly the tonoplast, as also revealed in yeast (Klionsky et al., 1990). ALPase activity has been used as a marker for metabolic activity of the fungi (Guillemin et al., 1995; Tisserant et al., 1993) because the enzyme was highly active in mature arbuscules (e.g. Fig. 1A), though the actual role of the enzyme has not been clarified (Larsen et al., 1996). Based on its 
substrate specificity it is unlikely that the ALPase can hydrolyze pyrophosphate compounds such as polyP and any suggestion of a direct role in polyP mobilization must be questioned. Recently, the involvement of ALPase in sugar metabolism has been suggested (Ezawa et al., 1999). AM fungi take up glucose in the symbiotic phase and metabolize it through glycolysis and/or the pentose phosphate pathway (Shachar-Hill et al., 1995; Solaiman and Saito, 1997), in which glucose 6-phosphate (glucose 6-P) is synthesized at the first step (Bago et al., 2000). Glucose 6-P is a key compound in sugar metabolism and is utilized in biosynthesis of various carbohydrates, including trehalose which is a temporary store of carbon in AM fungi (Bécard et al., 1991; Schubert et al., 1992). Glucose 6-P and trehalose 6-P (an intermediary metabolite in trehalose biosynthesis) were the most favorable substrates for the ALPase of AM fungi (Ezawa et al., 1999). In contrast, the ACPase of AM fungi showed no particular substrate specificity: not only phosphomonoesters but also pyrophosphate compounds can be hydrolyzed. The ALPase and ACPase, which have different $\mathrm{pH}$ optima and substrate specificities, may work together to liberate Pi from various P-esters over a wide range of vacuolar $\mathrm{pH}$.

\section{P transport along hyphae}

The forms of P involved in long distance transport along the hyphae of AM fungi are probably Pi and polyP, with the possible addition of organic $\mathrm{P}$ (ester-P). The size of vacuolar P pools in many organisms appears to respond flexibly to availability of $\mathrm{P}$ in the environment, with the vacuoles of plants (Bieleski, 1973) and yeast (Shirahama et al., 1996) acting as a Pi reservoir for the cytoplasm under P deficient conditions. Under relatively P-deficient conditions PolyP contributed between $5-17 \%$ of the total P in the hyphae of Gigaspora margarita (Solaiman et al., 1999). P-deficiency may accelerate $\mathrm{Pi}$ turnover in the vacuoles of $\mathrm{AM}$ fungi, resulting in low polyP/Pi or organic $\mathrm{P} / \mathrm{Pi}$ ratios.

Rapid changes in polyP content of AM hyphae in response to P supply have been observed. In Glomus coronatum polyP could not be detected at all in the extraradical hyphae of AM fungi if $\mathrm{P}$ 
was completely withheld one week before harvest, but when mycorrhizal plants were supplied with a solution containing $50 \mu \mathrm{M}$ Pi about $50 \%$ of the hyphae contained polyP (Ezawa et al., 2001a). Furthermore, Boddington and Dodd (1999) reported differences in the amount of polyP occurring in hyphae of different AM fungal species. This interesting observation requires to be confirmed, because the sensitivity and specificity of the stain used to visualize polyP (DAPI) has not yet been evaluated in filamentous fungi.

It has been suggested that the establishment of the symbiotic relationship enables AM fungi to take up and transport $\mathrm{Pi}$ in amounts beyond their own requirements, and that the vacuolar system may be responsible for the long distance transport of $\mathrm{P}$ along the hyphae. Motile tubular vacuoles have been demonstrated in a range of filamentous fungi (Rees et al., 1994) (see Ashford and Allaway, this volume; Bago et al., this volume). 'The moving vacuoles' can be observed in living cells, most clearly in actively growing young cultures, by loading fluorescent vacuolar tracers. In this system clusters of vacuoles are interconnected by tubules along which the vacuoles move (Hyde et al., 1997). The tubules are preserved in electron micrographs of freeze-substituted tissue but not in material fixed by conventional methods. $\mathrm{P}$ as well as $\mathrm{K}$ is distributed in the tubular vacuoles of the ectomycorrhizal fungus $P$. tinctorius, as revealed by energy-dispersive X-ray analysis (Cole et al., 1998). However, the role of the motile tubular vacuoles have not yet been fully established and we do not know if 'the moving vacuoles' labeled by fluorochrome also have $\mathrm{P}$ in the lumen or unique vacuolar enzymes such as V-ATPase. An analogous system to the Pisolithus motile tubular vacuoles has been observed in AM fungi (Uetake, personal communication; Dickson, personal communication), but has not yet been fully characterized. However, it is feasible that the system is involved in long distance P transport.

\section{Regulation of arbuscule formation}

Arbuscule formation is sensitive to $\mathrm{P}$ supply and a low to moderate supply is required. If the $\mathrm{P}$ supply is very low, colonization appears to be inhibited (Bolan et al., 1984). Without P fertilization 
Glomus intraradices and G. coronatum formed only intercellular hyphae but not arbuscules in the roots of tomato and mungbean, respectively, and the subsequent application of $\mathrm{P}$ triggered arbuscule formation (Rosewarne and Kasiamdari, personal communication). However, nutritionally adequate or high P supply tends to reduce both colonization and arbuscule formation, with the magnitude of the effect varying between plant species and also sensitive to change in environmental parameters such as irradiance. (reviewed in Smith and Read, 1997). These observations indicate that the symbionts, plant and/or fungus, are able to sense the P concentration in the environment and that this may lead to regulation of the expression of genes involved in arbuscule formation. This in turn would have an impact on P transfer to the plant.

\section{Regulation of Pi transfer via arbuscules}

The arbuscule has been recognized as the site from which Pi is almost certainly released to the interfacial apoplast and thence absorbed by the plant cortical cell. Supporting evidence includes: i) ATP-hydrolyzing activity, possibly P-ATPase, was localized on periarbuscular membrane of the host (Gianinazzi-Pearson et al., 1991) and ii) High-affinity Pi transporters (Rosewarne et al., 1999; Rausch et al., 2001) and P-ATPase (Gianinazzi-Pearson et al., 2000) genes are expressed in arbuscule-containing host cells. These observations suggest that $\mathrm{Pi}$ is actively taken up by the plant through the periarbuscular membrane. iii) ATP-hydrolyzing activity on the fungal plasma membrane of arbuscules is absent (Gianinazzi-Pearson et al., 1991) and iv) the fungal P transporter gene GvPT is not expressed within the roots (Harrison and Van Buuren, 1995). These observations suggest that active nutrient uptake does not occur in the fungal arbuscules.

Although the mechanism of Pi transfer from fungus to plant has not yet been elucidated, it is considered that it may be regulated at the following steps, i) arbuscule formation; ii) Pi liberation from the vacuolar P pool; and iii) efflux through the tonoplast and plasma membrane of AM fungi to the interfacial apoplast. There must be regulatory cross talk between the partners to prevent perturbation in Pi concentration in the fungal mycelium during the Pi transfer phase of arbuscular 
activity.

\section{Regulation of polyP turnover by V-ATPase}

During arbuscule formation, the balance between synthesis and hydrolysis of polyP may be altered via V-ATPase activity. Vacuolar $\mathrm{pH}$, influenced by activity of V-ATPases, may be a regulatory factor in polyP synthesis and hydrolysis. A V-ATPase-defective mutant of yeast is unable to acidify the vacuolar lumen and accumulate polyP (Beauvoit et al., 1991). Bafilomycin A1, a specific inhibitor of V-ATPase, increased vacuolar $\mathrm{pH}$ and induced polyP hydrolysis in yeast (Beauvoit et al., 1991). Furthermore, alkalinization of the vacuolar lumen, either by amine or protonophoric uncouplers, induces rapid hydrolysis of polyP in algae (Pick and Weiss, 1991) and yeast (Beauvoit et al., 1991). The rapidity of the response also implies that genes for polyP-hydrolyzing enzymes may be expressed constitutively. PPX/ACP activities in the intraradical hyphae of AM fungi were not affected by the absence or presence of polyP in the hyphae (Ezawa et al., 2001a). These observations suggest that polyP accumulation may be dynamically regulated by the balance between synthesis and hydrolysis. We suggest that the inactivation of V-ATPase may occur during maturation of arbuscules and inhibit synthesis of polyP, resulting in net hydrolysis of polyP.

The V-ATPase is a large and complex enzyme and consists of at least thirteen different types of subunit (Margolles-Clark et al., 1999). Post-transcriptional regulation of V-ATPase activity has been investigated extensively in yeast. The proton pumping activity is regulated by disassembly and reassembly of the cytoplasmic and membrane sectors of the enzyme in as little as $5 \mathrm{~min}$, without any new protein synthesis (Kane and Parra, 2000). The cytoplasmic sector could sense changes in ATP concentration or ATP/ADP ratio in the cytoplasm and regulate disassembly and reassembly of the enzyme. The ATP concentration and ATP/ADP ratios are the reflection of the energy status of a cell. In fact, glucose deprivation decreased the ATP level in cytosol of yeast and disassembled V-ATPase (Kane and Parra, 2000). These observations allow us to hypothesize the following scheme for AM fungal arbuscules: once arbuscule formation is initiated, the system involved in the 
delivery of the carbon source may be switched off [speculation supported by the absence of P-ATPase activity in arbuscules (Gianinazzi-Pearson et al., 1991; 2000)], and depletion of energy may disassemble the V-ATPase. Energy depletion may also inactivate the P-ATPase and promote Pi efflux via PD-sensitive channels on the fungal plasma membrane and probably also the tonoplast, due to depolarization of the membranes as observed in plants (Mimura, 1999). These events could lead to loss of cell function, but the fungi would be able to minimize the potential impact on the intraradical mycelium by forming a septum in the trunk hypha of each arbuscule (Dickson and Smith, 2001).

\section{Regulation of Pi efflux}

Solaiman and Saito (2001) investigated the efflux of Pi from isolated intraradical hyphae of Gigaspora margarita. They showed that glucose, which is probably the main carbon source of AM fungi, enhanced polyP hydrolysis and subsequent Pi efflux slightly, but significantly. They hypothesized that carbon uptake and Pi efflux in the fungus might be metabolically linked. On the other hand, Beauvoit et al. (1991) suggested that the polyP hydrolysis induced by glucose in yeast might be for buffering the cytoplasmic Pi concentration, which was decreased during glucose metabolism. The latter interpretation is more likely to fit the results of Solaiman and Saito (2001). However, their experiment provided an important and suggestive result in the context of the regulation of Pi efflux. Net hydrolysis of polyP was much slower than expected from the results of our enzymatic studies (Ezawa et al., 2001a). Maximal activity of polyP-hydrolyzing enzymes in the isolated hyphae of G. coronatum can be estimated as about $430 \mathrm{pmol} \mathrm{min}^{-1} \mathrm{mg}^{-1}$ hyphae from the following information: specific activity, 30 (soluble) and 25 (insoluble) $\mathrm{pmol} \mathrm{Pi} \mathrm{min}^{-1} \mu \mathrm{g}^{-1}$ protein at $\mathrm{pH}$ 5.0; protein concentrations per unit hyphae, 6 (soluble) and 10 (insoluble) $\mu \mathrm{g} \mathrm{mg}^{-1}$ hyphae; total activity, $30 \times 6+25 \times 10=430$. However, net Pi hydrolysis and efflux calculated from the data of Solaiman and Saito (2001) are only 13.4 and $16 \mathrm{pmol} \mathrm{min}^{-1} \mathrm{mg}^{-1}$ hyphae, respectively. If, as Solaiman and Saito (2001) point out, 'leakage' from the fragmented hyphae is taken into account, 
the true rate of efflux may be even slower. In their experiment, the majority of polyP (and probably organic P) was retained in the hyphae even after 4 hours incubation, so the 'slow hydrolysis' was not due to depletion of substrate. Of course these values are derived from different plant-fungal combinations. In addition, it is uncertain if the enzymes were fully accessible to the substrate and if the polyP synthetic pathway was still sufficiently active in the hyphae to reduce net hydrolysis of polyP. However, the fact that the net hydrolysis and efflux were about 30 times less than the predicted capacity of the hydrolytic enzymes strongly suggests that Pi efflux from the hyphae would be limited by activity of the Pi transporters/channels rather than activity of the enzymes. In general, activity of phosphatase-type enzymes could be physiologically controlled by Pi concentration i.e. a high concentration of end product $(\mathrm{Pi})$ inhibits phosphatases. This could be a mechanism by which $\mathrm{Pi}$ transporters/channels regulate hydrolysis of polyP/organic $\mathrm{P}$ in the vacuoles.

Information about transporters/channels involved in ion efflux from eukaryotic microorganisms is very limited. Researchers have probably not paid sufficient attention to anion efflux in fungi, because it may seem less important or interesting for understanding the basic system of fungal life. This is in contrast to plants in which anion efflux channels play significant roles in, for example, stomatal closure (Schroeder and Keller, 1992). So far Pi efflux in fungi has only been demonstrated for isolated yeast vacuoles. As described earlier, Booth and Guidotti (1997) demonstrated that the transporters/channels involved in Pi uptake by isolated vacuoles of yeast were actually driven by counterflow exchange with Pi present in the vacuoles. They found that two systems are involved in Pi efflux from the vacuoles: one was the bidirectional transport mediated by the transporters/channels, and the other was unidirectional transport which was much slower than the bidirectional system. Recently, an anion-selective efflux channel on the plasma membrane of a filamentous fungus, Aspergillus niger, has been characterized by the patch clamp technique in conjunction with an UV pulse laser as a tool for ablating the cell wall (Roberts et al., 1997). This technique may have great potential to investigate transporter activity of plant and fungal cells, particularly for those having cell walls that cannot easily be digested by commercial enzymes, such 
as AM fungi (Ezawa unpublished observation). Furthermore, the laser ablation technique enables selection of the site of patch clamp, so that localized transporter activity along hyphae can be measured. It is uncertain, however, whether the technique can be applied to the tips of finely branched arbuscules from which Pi efflux seems likely to occur.

\section{Conclusions}

A hypothetical mechanism of Pi efflux from arbuscules is summarized in Fig. 1. Validation of the hypothesis will require: i) identification of genes that are up- or down-regulated during arbuscule formation, ii) clarification of the role of V-ATPase activity in polyP synthesis/hydrolysis and its possible inactivation during arbuscule formation and iii) identification of factors which promote Pi efflux from arbuscules. To achieve these objectives some technical breakthroughs are required. Establishment of an experimental system in which frequency of arbuscules in the roots can be controlled by simple treatments such as P application is necessary to identify genes involved in arbuscule formation by the differential display or screening of cDNA libraries. Even if such genes are identified, we do not yet have a genetic manipulation technique to validate the function of the genes. The particle bombardment method is one possible way of inserting genes of interest into AM fungi, as shown by Forbes et al. (1998) who demonstrated transitory expression of a reporter gene in germinating spores of Gi. margarita. Future work should be directed at achieving expression in the symbiotic stages of fungal development, so that the function of genes of interest can be inhibited by introducing antisense genes. A technique for isolation of the intact vacuoles, or at least intact tonoplast, of the fungi must be established to measure V-ATPase activity and investigate the involvement of the enzyme in polyP metabolism. It has been accepted, as described earlier, that arbuscules are the site of Pi release, but it has not been proved directly i.e. P efflux from arbuscules must be visualized or hopefully measured quantitatively. Since the apparent rate of Pi efflux from the intraradical hyphae is slow on the basis of unit hyphal length (see previous section), a more sensitive and specific method to evaluate Pi efflux from arbuscules is required. 
Understanding $\mathrm{C}$ metabolism (see Bago et al., 2000) may be important to understand $\mathrm{P}$ metabolism. In the key steps of primary metabolism, $\mathrm{C}$ is metabolized via P-esters. Furthermore, the energy status of cell, which is a reflection of $\mathrm{C}$ metabolism, may influence membrane transport via $\mathrm{H}^{+}$-ATPases and other energy dependent reactions such as polyP synthesis. Thus it is possible that $\mathrm{C}$ metabolism may play a key role in the regulation of Pi transfer in the plant-fungus interface. Integration of the pictures of $\mathrm{P}$ and $\mathrm{C}$ metabolism may help us to understand a whole story of the mechanism of mutualistic relationship.

\section{Acknowledgements}

Our collaboration was supported by Japan Society for the Promotion of Science (Postdoctoral Fellowship to TE) and the Australian Research Council (SES and FAS).

\section{References}

Andreeva N A, Kulakovskaya T, Sidorov I, Karpov A and Kulaev I S 1998a Purification and properties of polyphosphatase from Saccharomyces cerevisiae cytosol. Yeast 14, 383-390.

Andreeva N A, Kulakovskaya T V and Kulaev I S 1998b Purification and properties of exopolyphosphatase isolated from Saccharomyces cerevisiae vacuoles. FEBS Letters 429, 194-196.

Andreeva N A and Okorokov L A 1993 Purification and characterization of highly active and stable polyphosphatase from Saccharomyces cerevisiae cell envelope. Yeast 9, 127-139.

Ayling S M, Smith S E and Smith F A 2000 Transmembrane electrical potential difference of germ tubes of arbuscular mycorrhizal fungi responds to external stimuli. New Phytol. 147, 631-639.

Bago B, Pfeffer PE, and Shachar-Hill Y 2000 Carbon metabolism and transport in arbuscular mycorrhizas. Plant Physiology 124, 949-958.

Beauvoit B, Rigoulet M, Raffard G, Canioni P and Guerin B 1991 Differential sensitivity of the cellular compartments of Saccharomyces cerevisiae to protonophoric uncoupler under 
fermentative and respiratory energy supply. Biochemistry 30, 11212-20.

Bécard G, Doner L W, Rolin D B, Douds D D and Pfeffer P E 1991 Identification and quantification of trehalose in vesicular-arbuscular mycorrhizal fungi by in vivo ${ }^{13} \mathrm{C}$ NMR and HPLC analyses. New Phytol. 118, 547-552.

Beever R E and Burns D J W 1980 Phosphorus uptake, storage and utilisation by fungi. Adv. Bot. Res. $8,127-219$.

Bieleski R L 1973 Phosphate pools, phosphate transport, and phosphate availability. Ann. Rev. Pl. Physiol. 24, 225-252.

Boddington C L and Dodd J C 1999 Evidence that differences in phosphate metabolism in mycorrhizas formed by species of Glomus and Gigaspora might be related to their life-cycle strategies. New Phytol. 142, 531-538.

Bolan N S, Robson A D and Barrow N J 1984 Increasing phosphorus supply can increase the infection of plant roots by vesicular-arbuscular mycorrhizal fungi. Soil Biol. Biochem. 16, 419-420.

Booth J W and Guidotti G 1995 An alleged yeast polyphosphate kinase is actually diadenosine-5',5"'-P-1,P-4-tetraphosphate $\alpha, \beta$-phosphorylase. J. Biol. Chem. 270, 19377-19382.

Booth J W and Guidotti G 1997 Phosphate transport in yeast vacuoles. J. Biol. Chem. 272, 20408-20413.

Bücking H and Heyser W 1999 Elemental composition and function of polyphosphates in ectomycorrhizal fungi - an X-ray microanalytical study. Mycol. Res. 103, 31-39.

Callow J A, Capaccio L C M, Parish G and Tinker P B 1978 Detection and estimation of polyphosphate in vesicular-arbuscular mycorrhizas. New Phytol. 80, 125-134.

Capaccio L C M and Callow J A 1982 The enzymes of polyphosphate metabolism in vesicular-arbuscular mycorrhizas. New Phytol. 91, 81--91.

Clark J E, Beegen H and Wood H G 1986 Isolation of intact chain of polyphosphate from 
Propionibacterium shermanii grown on glucose or lactate. J. Bacteriol. 168, 1212--1219.

Cole L, Orlovich D A and Ashford A E 1998 Structure, Function, and, Motility of Vacuoles in Filamentous Fungi. Fungal Genet. Biol. 24, 86-100.

Cox G, Moran K J, Sanders F, Nockolds C and Tinker P B 1980 Translocation and transfer of nutrients in vesicular-arbuscular mycorrhizas. New Phytol. 84, 649-659.

Cramer C and Davis R 1984 Polyphosphate-cation interaction in the amino acid-containing vacuole of Neurospora crassa. J. Biol. Chem. 259, 5152-5157.

Dickson S and Smith S E 2001 Cross walls in arbuscular trunk hyphae from after loss of metabolic activity. New Phytol. 151, 735-742.

Ezawa T, Kuwahara S, Sakamoto K, Yoshida T and Saito M 1999 Specific inhibitor and substrate specificity of alkaline phosphatase expressed in the symbiotic phase of the arbuscular mycorrhizal fungus, Glomus etunicatum. Mycologia 91, 636-641.

Ezawa T, Saito M and Yoshida T 1995 Comparison of phosphatase localization in the intraradical hyphae of arbuscular mycorrhizal fungi, Glomus spp. and Gigaspora spp. Pl. Soil 176, 57-63.

Ezawa T, Smith S E and Smith F A 2001a Differentiation of polyphosphate metabolism between the extra- and intraradical hyphae of arbuscular mycorrhizal fungi. New Phytol. 149, 555-563.

Ezawa T, Smith S E and Smith F A 2001b Enzyme activity involved in glucose phosphorylation in two arbuscular mycorrhizal fungi: indication that polyP is not the main phosphagen. Soil Biol. Biochem. 33, 1279-1281.

Fabig B, Moawad A M and Achtnich W 1989 Gas-chromatographic separation of organic acids and electrophorectic determination of phosphatases from VA mycorrhizal roots. Z. Pflanzernär. Bodenkd. 152, 261-265.

Forbes P J, Millam S, Hooker J E and Harrier L A 1998 Transformation of the arbuscular mycorrhiza Gigaspora rosea by particle bombardment. Mycol. Res. 102, 497-501.

Gianinazzi S, Gianinazzi-Pearson V and Dexheimer J 1979 Enzymatic studies on the metabolism of vesicular-arbuscular mycorrhiza. III. Ultrastructural localization of acid and alkaline 
phosphatase in onion roots infected by Glomus mosseae (Nicol. \& Gerd.). New Phytol. 82, $127--132$.

Gianinazzi-Pearson V, Arnould C, Oufattole M, Arango M and Gianinazzi S 2000 Differential activation of $\mathrm{H}^{+}$-ATPase genes by an arbuscular mycorrhizal fungus in root cells of transgenic tobacco. Planta 211, 609-613.

Gianinazzi-Pearson V and Gianinazzi S 1978 Enzymatic studies on the metabolism of vesicular-arbuscular mycorrhiza. II. Soluble alkaline phosphatase specific to mycorrhizal infection in onion roots. Physiol. Plant Pathol. 12, 45-53.

Gianinazzi-Pearson V, Smith S E, Gianinazzi S and Smith F A 1991 Enzymatic studies on the metabolism of vesicular-arbuscular mycorrhizas. V. Is $\mathrm{H}^{+}$-ATPase a component of ATP-hydrolysing enzyme activities in plant-fungus interfaces? New Phytol. 117, 61-74.

Guillemin J P, Orozco M O, Gianinazzi-Pearson V and Gianinazzi S 1995 Influence of phosphate fertilization on fungal alkaline phosphatase and succinate dehydrogenase activities in arbuscular mycorrhiza of soybean and pineapple. Agri. Ecosystem Environ. 53, 63-69.

Harrison M J and Van Buuren M L 1995 A phosphate transporter from the mycorrhizal fungus Glomus versiforme. Nature 378, 626-629.

Hyde G, Cole L and Ashford A E 1997 Mycorrhiza movies. Mycorrhiza 7, 167-169.

Kane P M and Parra K J 2000 Assembly and regulation of the yeast vacuolar $\mathrm{H}^{+}$-ATPase. J. Exp. Biol. 203, 81-87.

Klionsky D J, Herman P K and Emr S D 1990 The fungal vacuole, composition, function, and biogenesis. Microbiol. Rev. 54, 266-92.

Kornberg A, Rao N N and Ault-Riche D 1999 Inorganic polyphosphate, A molecule of many functions. Ann. Rev. Biochem. 68, 89-125.

Kulaev I S, Andreeva N A, Lichko L P and Kulakovskaya T V 1997 Comparison of exopolyphosphatases of different yeast cell compartments. Microbiol. Res. 152, 221-226. 
Kulaev I S and Kulakovskaya T V 2000 Polyphosphate and phosphate pump. Ann. Rev. Microbiol. $54,709-734$.

Kulakovskaya T V and Kulaev I S 1997 Transport of phosphate into vacuoles of Saccharomyces cerevisiae. Microbiologia 13, 71-74.

Kumble K D and Kornberg A 1996 Endopolyphosphatases for long chain inorganic polyphosphate in yeast and mammals. J. Biol. Chem. 271, 27146-27151.

Larsen J, Thingstrup I, Jacobsen I and Rosendahl S 1996 Benomyl inhibits phosphorus transport but not fungal alkaline phosphatase activity in a Glomus-cucumber symbiosis. New Phytol. 132, $127-133$

Lenburg M E and O'Shea E K 1996 Signaling phosphate starvation. Trends Biochem. Sci. 21, $383-7$.

Lichko L P, Kulakovskaya T V and Kulaev I S 1996 Characterization of the polyphosphatase activity of Saccharomyces cerevisiae nuclei. Biochemistry (Moscow) 61, 361-366.

Lichko L P, Kulakovskaya T V and Kulaev I S 1998 Membrane-bound and soluble polyphosphatases of mitochondria of Saccharomyces cerevisiae - identification and comparative characterization. Biochim. Biophys. Acta Biomembranes 1372, 153-162.

Margolles-Clark E, Tenney K, Bowman E J and Bowman B J 1999 The structure of the vacuolar ATPase in Neurospora crassa. J. Bioenergetics Biomembranes 31, 29-37.

Martinez P and Persson B L 1998 Identification, cloning and characterization of a derepressible $\mathrm{Na}^{+}$-coupled phosphate transporter in Saccharomyces cerevisiae. Mol. Gen. Genet. 258, 628-638.

Massonneau A, Martinoia E, Dietz K J and Mimura T 2000 Phosphate uptake across the tonoplast of intact vacuoles isolated from suspension-cultured cells of Catharanthus roseus (L.) G. Don. Planta 211, 390-395.

Mimura T 1999 Regulation of phosphate transport and homeostasis in plant cells. Inter. Rev. Cytol. 191, 149-200. 
Ogawa N, DeRisi J and Brown P O 2000 New components of a system for phosphate accumulation and polyphosphate metabolism in Saccharomyces cerevisiae revealed by genomic expression analysis. Mol. Biol. Cell 11, 4309-21.

Orlovich D A and Ashford A E 1993 Polyphosphate granules are an artefact of specimen preparation in the ectomycorrhizal fungus Pisolithus tinctorius. Protoplasma 173, 91-102.

Pick U and Weiss M 1991 Polyphosphate hydrolysis within acidic vacuoles in response to amine-induced alkaline stress in the halotolerant alga Dunaliella salina. Pl. Physiol. 97, $1234-1240$

Pisoni R and Lindley E 1992 Incorporation of $\left[{ }^{32} \mathrm{P}\right]$ orthophosphate into long chains of inorganic polyphosphate within lysosomes of human fibroblasts. J. Biol. Chem. 267, 3626-3631.

Rausch C, Daram P, Brunner S, Jansa J, Lalol M, Leggewie G, Amrhein N, and Bucher M 2001 A phosphate transporter expressed in arbuscule-containing cells in potato. Nature 414, 462-466.

Rees B, Shepherd V A and Ashford A E 1994 Presence of a Motile Tubular Vacuole System in Different Phyla of Fungi. Mycol. Res. 98, 985-992.

Reid R J, Mimura T, Ohsumi Y, Walker N A and Smith F A 2000 Phosphate uptake in Chara, Membrane transport via Na/Pi cotransport. Pl. Cell Environ. 23, 223-228.

Reizer J, Reizer A and Saier Milton H, Jr. 1994 A functional superfamily of sodium/solute symporters. Biochim. Biophys. Acta 1197, 133-166.

Roberts S K, Dixon G K, Dunbar S J and Sanders D 1997 Laser ablation of the cell wall and localized patch clamping of the plasma membrane in the filamentous fungus Aspergillus characterization of an anion-selective efflux channel. New Phytol. 137, 579-585.

Rosewarne G M, Barker S J, Smith S E, Smith F A and Schachtman D P 1999 A Lycopersicon esculentum phosphate transporter (LePT1) involved in phosphorus uptake from a vesicular-arbuscular mycorrhizal fungus. New Phytol. 144, 507-516.

Schroeder J I and Keller B U 1992 Two types of anion channel currents in guard cells with distinct voltage regulation. Proc. Natl. Acad. Sci. USA 89, 5025-5029. 
Schubert A, Wyss P and Wiemken A 1992 Occurrence of trehalose in vesicular-arbuscular mycorrhizal fungi and in mycorrhizal roots. J. Pl. Physiol. 140, 41-45.

Schweiger P F and Jakobsen I (1999). The role of mycorrhizas in plant P nutrition, fungal uptake kinetics and genotype variation. In Plant Nutrition - Molecular Biology and Genetics. Ed. G Gissel-Nielsen and A Jensen. pp 277-289. Kluwer Academic Publishers, Dordrecht.

Shachar-Hill Y, Pfeffer P E, Douds D, Osman S F, Doner L W and Ratcliffe R G 1995 Partitioning of intermediary carbon metabolism in vesicular-arbuscular mycorrhizal leek. P1. Physiol. 108, $7-15$.

Shirahama K, Yazaki Y, Sakano K, Wada Y and Ohsumi Y 1996 Vacuolar function in the phosphate homeostasis of the yeast Saccharomyces cerevisiae. Pl. Cell Physiol. 37, 1090-1093.

Smith S E and Read D J 1997 Mycorrhizal symbiosis. Academic Press. San Diego, California.

Solaiman M, Ezawa T, Kojima T and Saito M 1999 Polyphosphates in intraradical and extraradical hyphae of an arbuscular mycorrhizal fungus, Gigaspora margarita. Appl. Environ. Microbiol. $65,5604-5606$.

Solaiman M Z and Saito M 1997 Use of sugars by intraradical hyphae of arbuscular mycorrhizal fungi revealed by radiorespirometry. New Phytol. 136, 533-538.

Solaiman M Z and Saito M 2001 Phosphate efflux from the intraradical hyphae of an arbuscular mycorrhizal fungus, Gigaspora margarita, in vitro and its implication for phosphorus translocation in the hyphae. New Phytol. 151, in press.

Thomson B D, Clarkson D T and Brain P 1990 Kinetics of phosphorus uptake by the germ-tube of the vesicular-arbuscular mycorrhizal fungus Gigaspora margarita. New Phytol. 116, 647-654.

Tisserant B, Gianinazzi-Pearson V, Gianinazzi S and Gollote A 1993 In planta histochemical staining of fungal alkaline phosphatase activity for analysis of efficient arbuscular mycorrhizal infections. Mycol. Res. 97, 245-250.

Versaw W K and Metzenberg R L 1995 Repressible cation-phosphate symporters in Neurospora crassa. Proc. Natl. Acad. Sci. USA 92, 3884-3887. 
Wurst H and Kornberg A 1994 A soluble exopolyphosphatase of Saccharomyces cerevisiae purification and characterization. J. Biol. Chem. 269, 10996-11001.

Wurst H, Shiba T and Kornberg A 1995 The gene for a major exopolyphosphatase of Saccharomyces cerevisiae. J. Bacteriol. 177, 898-906.

Zhu Y-G, Cavagnaro T R, Smith S E and Dickson S 2001 Backseat driving? Accessing phosphate beyond the rhizosphere-depletion zone. Trend Pl. Sci. 6, 194-195. 


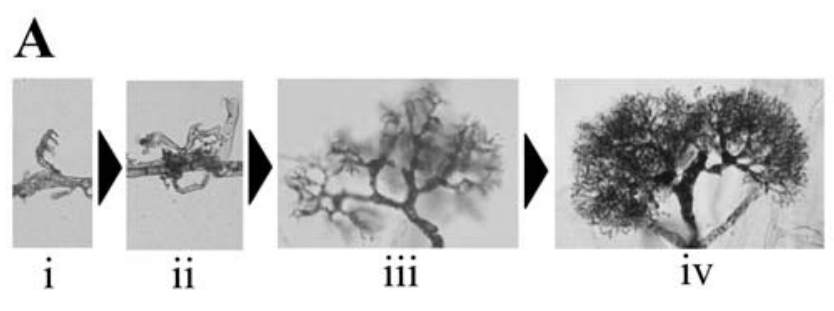

B
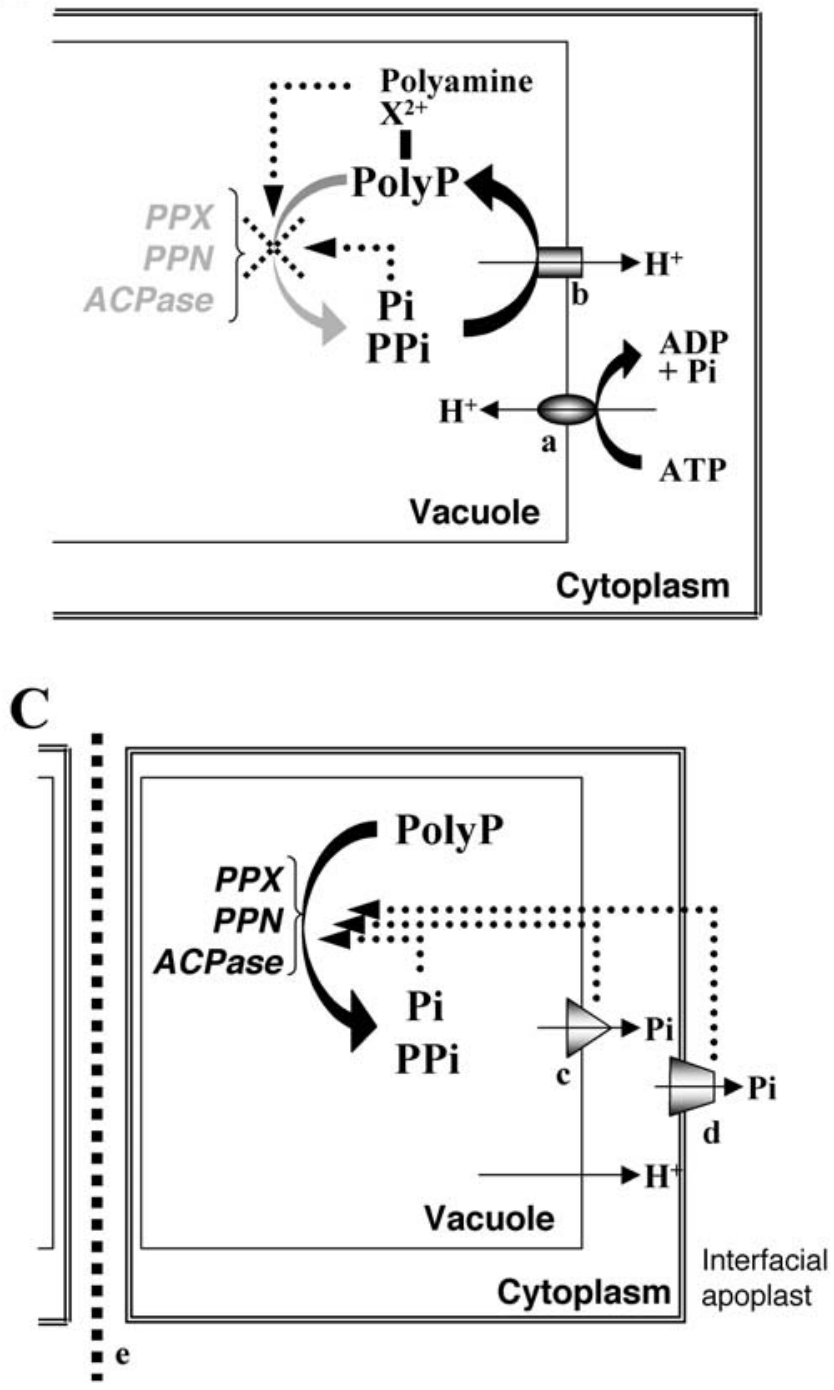

Fig. 1. Hypothetical scheme of Pi efflux from arbuscules. Efflux may be regulated at the following three steps, arbuscule formation, polyphosphate hydrolysis and Pi efflux through the membranes (see text). A, Development of an arbuscule from initiation to maturity i) The first trunk of arbuscule is initiated. ii) Two major branches are formed. iii) Fine branches are being formed. iv) Fully developed (mature) arbuscule showing high activity of alkaline phosphatase. B, Polyphosphate (polyP) metabolism in the initial phase of arbuscule formation. V-ATPase (a) may still be active and 
able to energize polyP synthesis (b). C, PolyP hydrolysis during maturation of an arbuscule. Inhibition of carbon delivery may inactivate V-ATPase and P-ATPase and net hydrolysis of polyP may occur. The depolarization of membranes due to inactivation of $\mathrm{H}^{+}$-ATPases may promote Pi efflux through potential difference (PD)-sensitive channels $(c, d)$ on the membranes. In this circumstance, the activity of Pi efflux transporters/channels may be the rate-limiting step. The fungi may minimize the impact by forming a septum (e) in the trunk of each arbuscule. $\mathrm{X}^{2+}$, divalent cations; PPX, exopolyphosphatase; PPN, endopolyphosphatase; ACPase, non-specific acid phosphatase. 